A new hybrid electrolyte VLE model is suggested. By extending the theoretical Debye-Hückel-Pitzer expression with the empirical Redlich-Kister term, a highly flexible model has been developed which is well suited for representing VLE behavior for specific systems. The system $\mathrm{H}_{2} \mathrm{O}-\mathrm{HCl}-\mathrm{MgCl}_{2}$ is accurately described in a temperature and concentration range of 273-423 $\mathrm{K}, 0-30$ molez $\mathrm{HCl}$ and $0-12$ moles MgCl2. 6 binary parameters (totally 11 when the temperature dependency is included) were used in the fit.

\title{
INTRODUCTION
}

Throughout this paper an electrolyte is interpreted as a mixture (solution) of ionic and molecular compounds. During the past 60 years several attemps have been made to obtain theoretical electrolyte activity models. All of these models are based upon the wellknown Debye-Hückel (1923) equation, which is commonly accepted as the "limiting law" expression for the activity of an ionic compound in a dielectric continum. However, due to the very complex nature of electrolytes, none of these general models are capable of handling concentrated solutions.

To get a satisfactory activity prediction in a concentrated solution, Chen (1980) and Pitzer (1980) suggests that an electrolyte should be treated with a hybrid equation, e.g. as a sum of a pure ionic and a molecular model. The contribution from the ionic term will dominate in the diluted region (here the electrolyte is almost completely dissociated) and the molecular term in the concentrated region. These kinds of equations have been shown to be very flexible.

\footnotetext{
Author to whom correspondence should be adressed. 0378-3812/83/\$03.00 @ 1983 Elsevier Science Publishers B.V,
} 
Chen (1980) used a hybrid model consisting of the Debye-Hückel equation and an NRTL model based upon all species in the electrolyte (ions and molecules). Pitzer (1980) used an extended DebyeHückel equation and a van Laar type model which only included the main components in the system (components in the sense of the phase rule). Pitzer's treatment seems to be more convenient for multicomponent mixtures because of the reduced number of components. This paper is to be regarded as an extension of Pitzer's work, with special attention to the thermodynamics behind hybrid models.

VLE IN ELECTROLYTES

From the basic equation for vapor-liquid equilibrium (1), the definition of activity (2) and fugacity (3) :

$\mu_{i}^{\ell}=\mu_{i}^{g}$

$\mu_{i}^{\ell}=\mu_{i}^{\star \ell}+\operatorname{RTIn} a_{i}$

$\mu_{i}^{g}=\mu_{i} \cdot g+\operatorname{RTln} f_{i} / f_{i}$

it is straightforward thermodynamics to establish the equilibrium criterion (4). Here the pressure dependency of the chemical potential is neglected.

$f_{i}=f_{i}^{\star} a_{i}$

The fugacity is commonly related to the pressure by introducing a fugacity coefficient:

$E_{i}=\varphi_{i} Y_{i} P$

There are several ways to relate the activity to the liquid composition, but in the case of electrolytes it is convenient to use a hypothetical mole fraction based upon a completely dissociated electrolyte. Let $i$ be any molecular and $j$ any ionic component in the solution and assume that $j$ dissociates according to the following equation:

$j=v+j++v-j-$

$v+$ are the number of cations $(j+)$, and $v-$ the number of anions

$(j-)$ in one formula unit of $j$. 
The activity of $i$ and $j$ may now be expressed in terms of the completely dissociated electrolyte mole fraction $(x)$ and activity coefficients :

$$
\begin{aligned}
& a_{i}=r_{i} x_{i} \\
& a_{j}=\left(\gamma_{j+j+} x_{j+}\right)^{v+}\left(r_{j-} x_{j-}\right)^{\nu-}=\left(\gamma_{j \pm} x_{j \pm}\right)^{\nu}
\end{aligned}
$$

$j \pm$ denotes the mean ionic properties, and $v=v++v-$. For any ion or molecule $k, x$ is related to the analytical mole fractions of $i$ and $j$ by:

$x_{k}=\frac{n_{k}}{\sum_{i}+\sum_{j} v n_{j}}$

One way to get an expression of the activity coefficients is to make a model of the excess Gibbs free energy for the mixture:

$\Delta G^{E}=G^{\text {real mix }}-G^{\text {ideal mix }}$

Partial differentiation with respect to a molecular component yields the activity coefficient according to eqn. (7):

$\left(\frac{\partial \Delta G^{E}}{\partial n_{i}}\right)_{P, T, n_{k}}=R T I n \gamma_{i}$

Partial differentiation with respect to an ionic compound yields the mean ionic activity coefficient according to eqn. (8):

$\left(\frac{\partial \Delta G^{E}}{\partial n_{j}}\right)_{P, T, n_{k}}=R T \ln \gamma_{j+}^{\nu+}+R T \ln \gamma_{j-}^{\nu-}=R T \ln \gamma_{j \pm}^{\nu}$

Equation (8) and (12) give the relationship between the fictitious ionic activity coefficients $(\gamma+, \gamma-)$ and the observable mean activity coefficient $\gamma+$. Although $\gamma+$ and $\gamma$ - are physically unrealistic, they play an important role in the electrolyte theory. This is due to the fact that every electrolyte is completely dissociated at infite dilution, and in this case the true mole fractions of the free ions $\left(x_{j^{+}}\right.$and $\left.x_{j_{-}}\right)$may be calculated according to eqn. (9). Knowing the concentrations of the free ions it is possible to develop a theoretical activity expression by means of statistical thermodynamics. These kinds of equations may give the ionic activities although they are not physically measurable. 
In concentrated solutions the theoretical activity expressions will, however, fail to work because of significant ion - ion interaction, and some kind of correction has to be used. In our opinion the best way to make this correction is to express the mean ionic activity coefficlent $\left(\gamma_{j \underline{\mu}}\right)$ in terms of $x_{j}$ without any knowledge of the true values of $x_{j+}$ or $x_{j-\cdot}$. This leads to the mixing of two excess-functions, one in terms of $x$ and one in terms of $x$. The way this problem may be solved is to combine eqns. (10) and (12):

$$
\left(\frac{\partial \Delta G^{E}}{\partial n_{j}}\right)_{P, T, n_{i}}=v+\left(\frac{\partial \Delta G^{E}}{\partial n_{j+}}\right) P_{, T, n_{i}}+v-\left(\frac{\partial \Delta G^{E}}{\partial n_{j-}}\right) P, T, n_{i}
$$

The last equation is very important because it utilizes a direct mixing of a theoretical ionic model in terms of $x_{i}, x_{j+}, x_{j-}$ and a molecular model in terms of $x_{i}, x_{j}$.

PROPOSED ELECTROLYTE MODEL

To represent the activity coefficients in electrolyte solutions we propose to use a hybrid model consisting of the Debye-HückelPitzer (DHP) equation for the electrostatic forces (Pitzer, 1980), and the Redich-Kister (RK) 3 parameter model for the residual part (Redlich and Kister, 1948). This combination is justified by the simple form of DHP and the great flexibilty of RK. The excess expression now becomes (pure components as reference states):

$$
\begin{aligned}
\frac{\Delta G^{E}}{R T} & =\left(\sum_{i} n_{i}+\sum_{j} v_{j} n_{j}\right)\left(\frac{1000}{M}\right) 1 / 2 \frac{4 A_{j} \ln 10}{3 \rho} I I n\left(1+\rho I^{1 / 2}\right) \\
& +\sum_{i} n_{i}\left(\sum \sum_{i} x_{i} x_{k}\left(b_{i k}+c_{i k}\left(x_{i}-x_{k}\right)+a_{i k}\left(x_{i}-x_{k}\right)^{2}\right) / 2\right) \\
& -\sum_{j} v_{j} n_{j} R T \ln \gamma_{j \pm, x_{j}=1}
\end{aligned}
$$

In the summations above, $j$ may be any ionic component and $i, k$ any ionic or molecular component.

VLE IN THE SYSTEM $\mathrm{H}_{2} \mathrm{O}-\mathrm{HCl}-\mathrm{MgCl}_{2}$

In most of the temperature range of interest, this system consist of one subcritical $\left(1-\mathrm{H}_{2} \mathrm{O}\right)$, one supercritical $(2-\mathrm{HCl})$, and one solid component $\left(3-\mathrm{MgCl}_{2}\right)$. 
The choice of suitable reference states then becomes quite difficult, but it appears to be convenient to use pure $\mathrm{H}_{2} \mathrm{O}, \mathrm{HCl}$ in azeotroplc $\mathrm{H}_{2} \mathrm{O}-\mathrm{HCl}$ mixture (az), and an infinite dilute solution of $\mathrm{MgCl}_{2}$ in $\mathrm{H}_{2} \mathrm{O}(\infty)$ as reference solutions for the 3 components respectively. Since $\mathrm{MgCl}_{2}$ is nonvolatile the VLE criterion now becomes:

$\varphi_{1} \mathrm{y}_{1} P=\gamma_{1} \mathrm{X}_{1} \varphi_{1}^{*}{ }^{*}{ }_{1}^{*}$

$\varphi_{2}{ }_{2}{ }^{P}=r_{2+}^{2} x_{2 \pm}^{2}{ }_{2}^{\varphi}{ }_{2}^{*}{ }_{2}^{*} P_{2}^{*}$

Normalizing the activity coefficient of $\mathrm{HCl}$ to 1 in an azeotropic solution of $\mathrm{H}_{2} \mathrm{O}-\mathrm{HCl}$ fixes the hypothetical reference state of $\mathrm{HCl}$ :

${ }^{*}{ }_{2}^{*}{ }_{2}^{*}{ }_{2}^{*}{ }_{2}^{*}=\frac{\varphi}{{ }_{2, a z} Y_{2, a z}{ }^{P} a z}$

By introducing the azeotropic mole fraction of $\mathrm{HCl}$ the final VLE expression for $\mathrm{HCl}$ becomes:

$\varphi_{2} y_{2} P=\varphi_{2, a z} \gamma_{2 \pm}^{2} \chi_{2 \pm}^{2} \frac{\left(1+x_{2, a z}\right)^{2}}{x_{2, a z}} P_{a z}$

Vapor phase fugacities are calculated from the second virial coefficients given by Wozny (1979). The following $\mathrm{H}_{2} \mathrm{O}$ vapor pressure and $\mathrm{H}_{2} \mathrm{O}-\mathrm{HCl}$ azeotropic data have been used:

$$
\begin{aligned}
& \operatorname{lnP_{1}^{*}}=77.78-\frac{7376}{T}+.007044 \mathrm{~T}-9.072 \ln (\mathrm{T}) \\
& \operatorname{lnP_{az}}=-225.8+\frac{1060}{\mathrm{~T}}-.06964 \mathrm{~T}+43.161 \mathrm{ln}(\mathrm{T}) \\
& \mathrm{x}_{2, \mathrm{az}}=.1325+.002941 \mathrm{~T}-.9151 \cdot 10^{-6 \mathrm{~T}}{ }^{2}
\end{aligned}
$$

By introducing eqn. (13) in (14) the normalized activity coefficients of $\mathrm{H}_{2} \mathrm{O}, \mathrm{HCl}$ and $\mathrm{MgCl}_{2}$ may be written as:

$$
\begin{aligned}
\ln \gamma_{1} & =\ln \gamma_{1}^{D H P}+\ln \gamma_{1}^{R K} \\
\ln \gamma_{2 \pm}^{2} & =\left(\ln \gamma_{2+}+\ln \gamma_{2-}-\ln \gamma_{2+, a z}-\ln \gamma_{2-, a z}\right) D H P \\
& +\left(\ln \gamma_{2 \pm}^{2}-\ln \gamma_{2 \pm, a z}^{2}\right)^{R K} \\
\ln \gamma_{3 \pm}^{3} & =\left(\ln \gamma_{3+}+2 \ln \gamma_{3-}^{2}\right)^{D H P}+\left(\ln \gamma_{3 \pm}^{3}-1 n \gamma_{3 \pm, \infty}^{3}\right) R
\end{aligned}
$$


The final activity coefficient expressions are given in appendix 1. Comparison of reported and calculated isotherms is shown in Figs. I and 2. The model has three adjustable Redich-Kister parameters for each binary; the two lower-order parameters (bij and cij) were assumed to be linear functions of temperature. The resulting 15 parameters were found by a regression analysis of experimental $x, Y, P, T$ data. Due to the limited concentration range, 4 of these parameters were found to be insignificant and therefore fixed equal to 0 . The optimal parameters are listed below:

$b_{12}=-.636+.222 \mathrm{~T}, c_{12}=-51.699-.0505 \mathrm{~T}, \mathrm{~d}_{12}=24.25$

$b_{13}=1001.315+1.955 \mathrm{~T}, c_{13}=313.702-.6340 \mathrm{~T}, d_{13}=0.00$

$b_{23}=-287.914+.625 \mathrm{~T}, c_{23}=0.000-.0000 \mathrm{~T}, \mathrm{~d}_{23}=0.00$

The model has been tested thoroughly against all available literature data, and the range of validity seems to be $273-423 \mathrm{~K}$, 0-30 molez $\mathrm{HCl}$ and $0-12$ molef $\mathrm{MgCl}_{2}$. The calculated phase diagram for the system $\mathrm{H}_{2} \mathrm{O}-\mathrm{HCl}-\mathrm{MgCl}_{2}$ is shown in Fig. 3 .

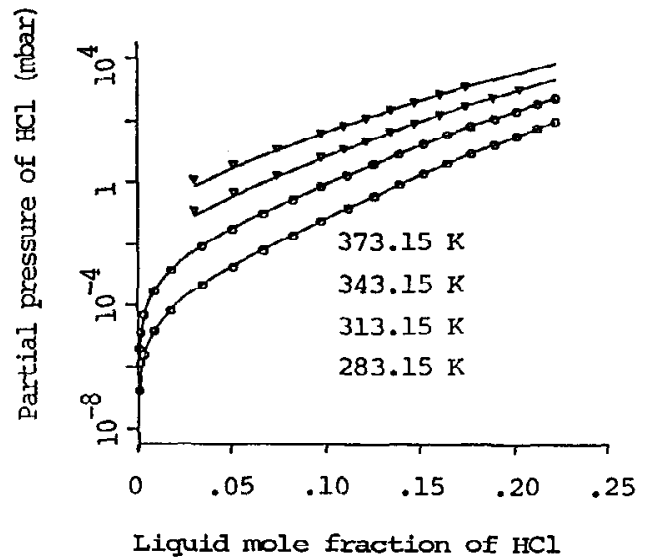

Liquid mole fraction of $\mathrm{HCl}$

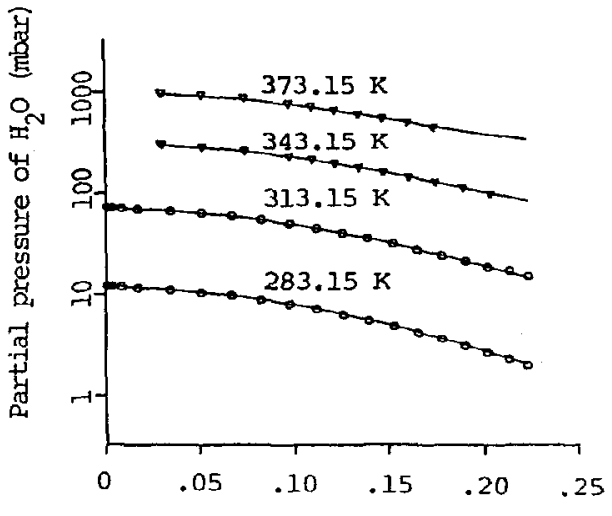

Liquid mole fraction of $\mathrm{HCl}$

Fig.1. Data fitting in the system $\mathrm{H}_{2} \mathrm{O}-\mathrm{HCl}$. o experimental data from Fritz and Fuget (1956). $\nabla$ experimental data from Perry (1975). Full lines are calculated values. 


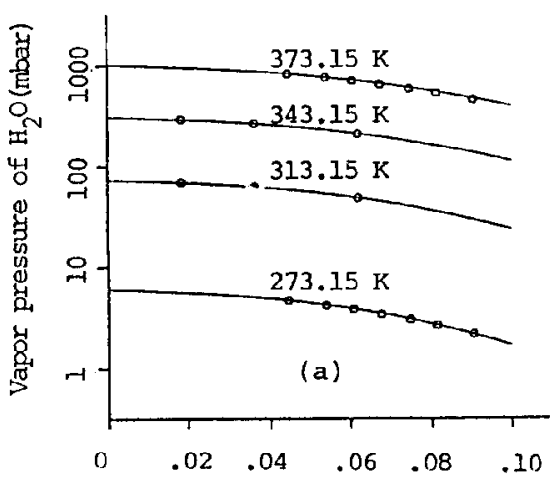

Liquid mole fraction of $\mathrm{MgCl}_{2}$

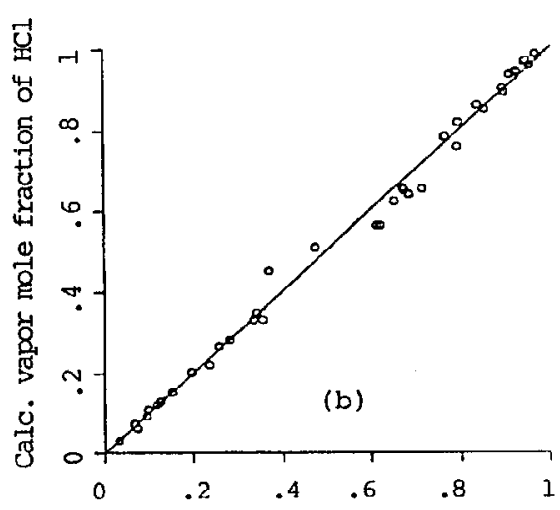

Exp. vapor mole fraction of $\mathrm{HCl}$

Fig.2. Data fitting in the system $\mathrm{H}_{2} \mathrm{O}-\mathrm{MgCl}_{2}$ (a) and $\mathrm{H}_{2} \mathrm{O}-\mathrm{HCl}^{-} \mathrm{MgCl}_{2}$ (b). O experimental values from Gmelin (1939) and Mirsch (1958) respectively. Full lines are calculated values.

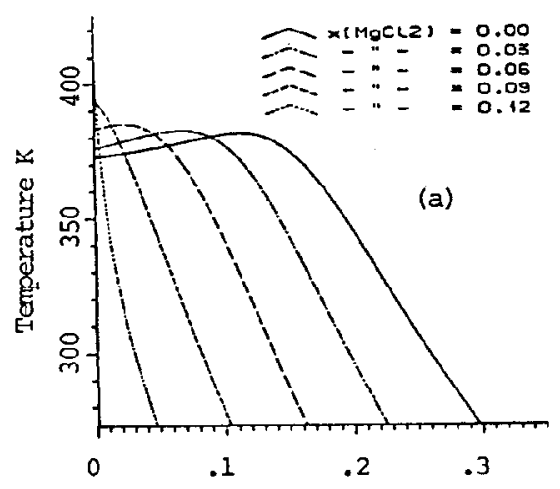

Liquid mole fraction of $\mathrm{HCl}$

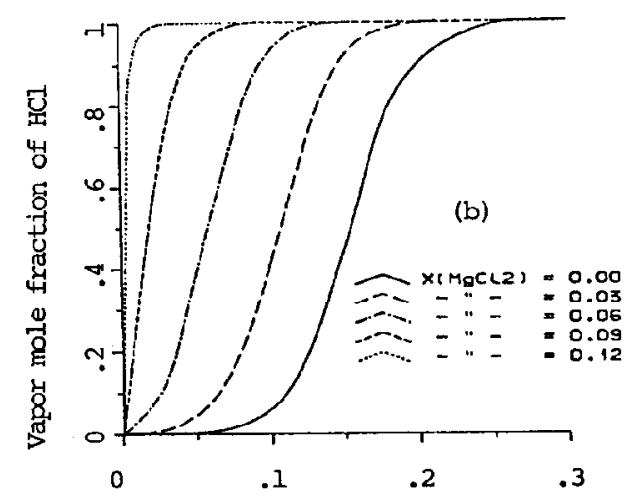

Liquid mole fraction of $\mathrm{HCl}$

Fig.3. Calculated bubble point curves (a) and the corresponding $\mathrm{xy}$ - diagram (b) for the system $\mathrm{H}_{2} \mathrm{O}-\mathrm{HCl}_{-} \mathrm{MgCl}_{2}$ at $1000 \mathrm{mbar}$. 
PARTIAL MOLAR ENTHALPIES FOR THE SYSTEM $\mathrm{H}_{2} \mathrm{O}-\mathrm{HCl}-\mathrm{MgCl}_{2}$

The use of classical thermodynamics give the relationship between activity coefficients and partial molar enthalpies in the liquid:

$$
\left(\frac{\partial \ln \gamma_{k}}{\partial T}\right)_{P, x}=-\frac{\bar{h}_{k}-\bar{h}_{k}^{*}}{R T^{2}}
$$

Enthalpies have been predicted from the previously described VLE model (which was based on VLE data only) to check the temperature dependency of the activity coefficients. Comparison of reported and calculated enthalpies are shown in Fig. 4. The agreement is very good.



Liquid mole fraction of $\mathrm{MgCl}_{2}$

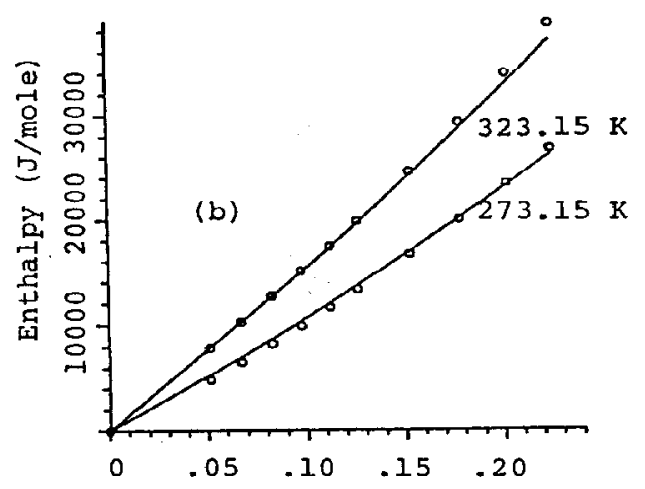

Iiquid mole fraction of $\mathrm{HCl}$

Fig.4. Partial molar enthalpy of (a) $\mathrm{H}_{2} \mathrm{O}$ in $\mathrm{H}_{2} \mathrm{O}-\mathrm{MgCl} \mathrm{Cl}_{2}$ and (b) $\mathrm{HCl}$ in $\mathrm{H}_{2} \mathrm{O}-\mathrm{HCl}$. o experimental data from Gmelin (1939). Full Iines are values predicted from the VLE model.

\section{CONCLUS ION}

A new semiempirical, hybrld, electrolyte activity model has been developed and successfully applied to the system $\mathrm{H}_{2} \mathrm{O}-\mathrm{HCl}_{-1} \mathrm{MCl}_{2} \cdot \mathrm{The}$ model is highly flexible, and should be well suited for industrial applications where the objective often is to get a good fit for a specific system. The model is not a general predictive one, and its use must be based on experimental data. The model has a sound physical basis, however, and safe extrapolations may be made to some extent beyond the range of experimental data. 
APPENDIX 1

Activity coefficient of component $k$ according to eqn. (14) and (22)-(23). (i,j and $k$ may be any ionic or molecular component $)$ :

$$
\begin{aligned}
& \operatorname{ln\gamma _{k}}=-\left(\frac{1000}{M_{s}}\right) 1 / 2 \frac{A_{\gamma} \ln 10}{3}\left(\frac{2 v z_{k \pm}^{2}}{\rho} \ln \left(1+\rho I^{1 / 2}\right)+\frac{v z_{k \pm}^{2} I^{1 / 2}-2 v I^{3 / 2}}{1+\rho I^{I / 2}}\right) \\
& +\sum_{j=1}^{N} x_{j}\left(b_{k j}+c_{k j}\left(2 x_{k}-x_{j}\right)+d_{k j}\left(x_{k}-x_{j}\right)\left(3 x_{k}-x_{j}\right)\right) \\
& -\sum_{i=1}^{N} \sum_{j=1}^{N} \frac{1}{2} x_{i} x_{j}\left(b_{i j}+2 c_{i j}\left(x_{i}-x_{j}\right)+3 a_{i j}\left(x_{i}-x_{j}\right)^{2}\right) \\
& -\ln _{k, x_{k}}=x_{\text {norm }} \\
& v z_{k \pm}^{2}=v_{k+} z_{k+}^{2}+v_{k-} z_{k-}^{2} \quad \text { (=0 for molecular components) } \\
& A_{Y}=\frac{\left(2 \pi N_{0} d_{s}\right)^{1 / 2}}{\ln 10}\left(\frac{e^{2}}{\varepsilon_{0} D k T}\right)^{3 / 2}=57708.995\left(\frac{d_{s}^{1 / 3}}{D T}\right)^{3 / 2} \\
& I \quad=\frac{1}{2} \sum_{k}\left(z_{k-}^{2} x_{k-}+z_{k+}^{2} x_{k+}\right)
\end{aligned}
$$

\section{LIST OF SYMBOLS}

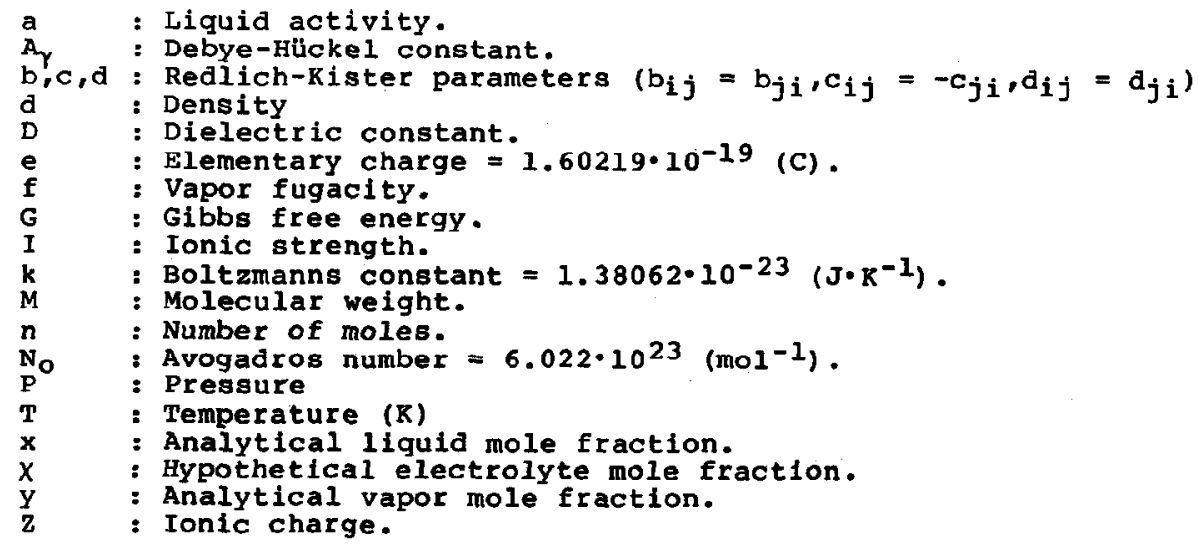




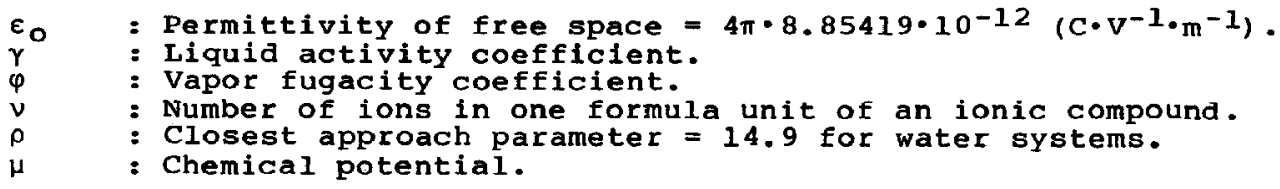

\section{SUPERSCRIPT}



\section{SUBSCRIPT}

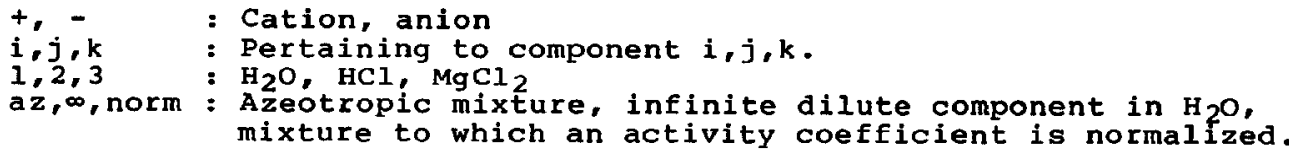

\section{REF ERENCES}

Chen, C.C., 1980. Computer simulation of Chemical Processes with Electrolytes, D.Sc.. Massachucetts Institute of Technology. Fritz, J.J. and C.R. Fuget, 1956. Ind.Eng.Chem.Data.Ser., 1: 10-12. Gmelins Handbuch der Anorganischen Chemie, 1939. Syst. nr. 27 B. 114-29.

Mirsch, E., 1958. Chem. Tech., 10: 540-41.

Mirsch, E. and M. Tittel, 1958. Chem. Tech., 10: 539-40.

Perry, R.H. and C.H. Chilton, 1975. Chemical Engineer's Handbook, 5th.ed.. McGraw-Hill Kogakusha LTD., New York.

Pitzer, K.S., 1980. J. of. Am. Chem. Soc., 102: 2902-06.

Redlich, O. and A.T. Kister, 1948. Ind. Eng. Chem., 40: $345-48$.

Wozny, G., 1979. Phasengleichgewichte starker Electrolyte aus Dampfaruckmessungen und deren Anwendung zur Auslegung von Trennkolonnen bei Thermochemischen Wasserzersetzungprozessen, Dr.Ing.. RWTH Aachen,. 